\title{
Starting a Fixed-Dose Combination Pill versus a Single Agent for Patients with Hypertension
}

\author{
Edward J. Filippone, MD, FACP' and Andrew J. Foy, MD \\ 'Sydney Kimmel Medical College at Thomas Jefferson University, Philadelphia, PA, USA; ${ }^{2}$ Penn State Milton S. Hershey Medical Center and College \\ of Medicine, Hershey, PA, USA.
}

J Gen Intern Med 32(6):597-8

DOI: $10.1007 / \mathrm{s} 11606-017-4013-2$

(c) Society of General Internal Medicine 2017

$\mathrm{H}$ ypertension remains the number-one cause of morbidity and mortality worldwide, ${ }^{1}$ and it is well established that treating elevated blood pressure with antihypertensive medications improves these outcomes. ${ }^{2}$ Target blood pressure values have been promulgated by societies in both Europe and the United States, although large surveys indicate that a significant percentage of patients treated for hypertension do not reach these targets. ${ }^{3}$ It is now recommended that therapy be initiated with two rather than one medication in individuals with higher pretreatment blood pressure in order to enhance the chances of success. ${ }^{4,5}$ Overall, the most commonly used classes of antihypertensives (i.e., diuretics, beta blockers [BB], calcium channel blockers [CCB], and renin-angiotensin system inhibitors [RASI]) are approximately equally effective in reducing cardiovascular events and mortality. ${ }^{2}$

No medication can produce beneficial effects, however, if the patient doesn't take it. Therapeutic persistence refers to the continuation of any antihypertensive therapy at some future point (e.g., 1 or 3 years) with at least one agent, while adherence (or compliance) indicates the percentage of days the patient actually takes a medication $(\geq 80 \%$ of days indicating full adherence). Most studies looking at these measures find woefully poor persistence and adherence, ${ }^{6,7}$ which certainly represents a major reason that hypertension is not effectively controlled overall.

Various reasons have been proposed to explain the lack of persistence and adherence, including side effects, frustration, cost, excessive pill burden, and lack of understanding of the necessity of treatment. Both European and American societies recommend fixed-dose combination (FDC) therapy as a viable alternative in order to reduce pill burden and improve adherence. ${ }^{4,5} \mathrm{FDC}$ treatment has been repeatedly shown to improve persistence/ adherence in comparison to each component taken as a separate pill, ${ }^{8,9}$ although lack of adherence to a single-pill FDC results in the simultaneous loss of the effect of both medications.

If two medications are indicated at the initiation of treatment, FDC therapy has obvious advantages in terms of pill

Published online March 8, 2017 burden and regimen simplicity. What is not clear is whether an FDC has advantages over a single agent and should be considered for all patients at the initiation of hypertension treatment as a means of enhancing persistence and adherence.

In an effort to answer this question, Lauffenburger et al. performed a retrospective analysis using administrative claims over a 5-year period from 484,493 patients who initiated treatment with antihypertensive medications. This study, published in this issue of the Journal of General Internal Medicine,$^{10}$ is by far the largest in this area. The authors compared persistence and full adherence at 1 year between 383,269 patients who began monotherapy and 78,958 patients who began FDC treatment. The 22,266 patients who initiated therapy with multiple separate pills were also considered. In multivariable analysis, persistence was increased with FDC by $9 \%$ (relative risk [RR] 1.09, 95\% CI 1.08-1.10) and adherence by $13 \%$ (RR 1.13, 1.11-1.14) compared to monotherapy. Surprisingly, adherence and compliance were greater with multiple separate pills than with an FDC. The authors conclude that FDC should be preferred to monotherapy when initiating antihypertensive therapy. We feel it is premature to make this recommendation, based on this and other studies.

Grimmsmann and Himmel retrospectively evaluated antihypertensive therapy persistence over 4 years in 8032 patients who initiated monotherapy (6502), FDCs (867), or free twodrug combination therapy (663). ${ }^{11}$ Persistence was best with free two-drug combinations (56.4\%) compared to single-pill therapy, whether monotherapy $(40.3 \%)$ or FDC (39.8\%). In contrast to the results reported by Lauffenburger et al., persistence was nearly the same for single-pill therapy whether it was monotherapy or an FDC. Similar to Lauffenburger et al.'s study, however, persistence was best with two free drugs. In contrast to these two studies, meta-analyses of prior studies have revealed better persistence/adherence with FDC than with two-drug combinations, although monotherapy was not evaluated in these studies. ${ }^{8,9}$ Hence, the literature provides conflicting evidence of whether it is optimal, purely from a persistence/adherence perspective, to initiate antihypertensive therapy with single-pill monotherapy, an FDC, or two free drugs.

In support of the position of Lauffenburger et al., there are several potential reasons that an FDC may result in better persistence/adherence than monotherapy. First, side effects may be lessened, such as a reduction in edema when combining 
an RAS blocker with CCB or reduced hyperkalemia by adding a diuretic to a RASI. Also, lower doses of each medication in an FDC may be required than with either medication as monotherapy, which may reduce side effects. ${ }^{12}$ Second, there may be less need for adding a second medication or otherwise altering therapy to reach target pressure, and hence less "therapeutic turbulence." Therapy modifications of any type have been associated with reduced adherence. ${ }^{6}$ Finally, overall pill burden may be reduced if one of the medications in the FDC would otherwise be prescribed for non-pressure-related indications, such as RAS blockade for heart failure or proteinuria.

There are several concerns with the Lauffenburger et al. study, however, that must be stressed and that prevent application of their data as justification for changing practice in the initial treatment of hypertension. First, the indications for therapy in their study are unknown, and other studies have shown that in as many as $27 \%$ of patients prescribed antihypertensive agents, hypertension is not the reason for treatment. ${ }^{7}$ It is possible that patients prescribed an FDC were more often being treated for HTN than were those started on a single agent. Obvious examples include BB for patients following a heart attack, non-dihydropyridine $\mathrm{CCB}$ for rate control of atrial fibrillation, and RASI for congestive heart failure or proteinuria. In their study, only $47 \%$ had a diagnosis of hypertension in the 180 days prior to initiation of therapy.

Second, it has been repeatedly shown that there are differences in persistence/adherence with both monotherapy and FDC based on the individual agents involved. ${ }^{6,7}$ RASIs, alone or in combination, have the highest persistence/adherence, and we would expect an FDC containing a RASI to have better persistence/ adherence than monotherapy with a BB or CCB. A fairer comparison, in our opinion, would be between monotherapy with a given medication and an FDC containing that medication.

Third, there is no mention of effectiveness in terms of blood pressure response at the end of therapy. If there was no difference despite lower persistence/adherence with a single drug, it could be argued that some of the patients on monotherapy may have initially had a lesser degree of blood pressure elevation, and may have had transient elevations that did not require long-term therapy. Additionally, there are no data on morbidity and mortality, which are the most important outcomes on which to base recommendations.

Finally, their multivariable analyses did not account for center effects, a potential source of bias. Some physicians/ practices may routinely initiate therapy with an FDC and have a population more likely to remain persistent/adherent based on factors such as socioeconomic status and level of education.

In conclusion, Lauffenburger et al. are to be commended for performing this provocative study with such important implications. Given the poor track record of persistence and adherence with drug treatment of hypertension, the number-one cause of mortality worldwide, any positive measure is most welcome. There is no question that a large multicenter randomized controlled trial is necessary to assess the most effective way to initiate therapy for hypertension, be it one drug, two individual drugs, or an FDC. Such a study should be large enough and long enough to compare blood pressure response and hard outcomes, including cardiovascular morbidity and mortality. Persistence and adherence could be assessed at various points along the way. Based on the available data, at this time we do not think it advisable to automatically start all patients with hypertension on an FDC.

Corresponding Author: Andrew J. Foy, MD; Penn State Milton S. Hershey Medical Center and College of Medicine, Hershey, PA, USA (e-mail: afoy@pennstatehealth.psu.edu).

\section{Compliance with Ethical Standards:}

Conflict of Interest: The authors do not have any financial conflicts of interest to disclose.

\section{REFERENCES}

1. Ezzati M, Lopez AD, Rodgers A, Vander Hoorn S, Murray CJ. Selected major risk factors and global and regional burden of disease. Lancet. 2002;360(9343):1347-1360. doi:10.1016/S0140-6736(02)11403-6.

2. Turnbull F. Blood Pressure Lowering Treatment Trialists' Collaboration. Effects of different blood-pressure-lowering regimens on major cardiovascular events: Results of prospectively-designed overviews of randomised trials. Lancet. 2003;362(9395):1527-1535.

3. Wolf-Maier K, Cooper RS, Kramer H, et al. Hypertension treatment and control in five European countries, Canada, and the United States. Hypertension. 2003;43(1):10. http://hyper.ahajournals.org/content/ 43/1/10.abstract.

4. Mancia G, Fagard R, Narkiewicz K, et al. ESH/ESC guidelines for the management of arterial hypertension: The task force for the management of arterial hypertension of the European Society of Hypertension (ESH) and of the European Society of Cardiology (ESC). Eur Heart J. 2013;34(28):2159-2219. doi:10.1093/eurheartj/eht151.

5. Weber MA, Schiffrin EL, White WB, et al. Clinical practice guidelines for the management of hypertension in the community a statement by the American Society of Hypertension and the International Society of Hypertension. J Hypertens. 2014;32(1):3-15. doi:10.1097/HJH. 0000000000000065

6. Caro JJ, Speckman JL, Salas M, Raggio G, Jackson JD. Effect of initial drug choice on persistence with antihypertensive therapy: the importance of actual practice data. CMAJ. 1999;160(1):41-46. Accessed 12 January 2017.

7. Poluzzi E, Strahinja P, Vargiu A, et al. Initial treatment of hypertension and adherence to therapy in general practice in Italy. Eur $\mathrm{J}$ Clin Pharmacol. 2005;61(8):603-609. doi:10.1007/s00228-005-0957-y. Accessed 12 January 2017.

8. Bangalore S, Kamalakkannan G, Parkar S, Messerli FH. Fixed-dose combinations improve medication compliance: a meta-analysis. Am J Med. 2007;120(8):713-719. doi:10.1016/j.amjmed.2006.08.033.

9. Gupta AK, Arshad S, Poulter NR. Compliance, safety, and effectiveness of fixed-dose combinations of antihypertensive agents. Hypertension. 2010;55(2):399. http://hyper.ahajournals.org/content/55/2/399.abstract.

10. Lauffenburger JC, Landon JE, Fischer MA. Effect of combination therapy on adherence among US patients initiating therapy for hypertension: a cohort study. J Gen Intern Med. 2017. doi:10.1007/s11606016-3972-z.

11. Grimmsmann T, Himmel W. Comparison of therapy persistence for fixed versus free combination antihypertensives: a retrospective cohort study. BMJ Open. 2016;6(11). http://bmjopen.bmj.com/content/6/11/ e011650.abstract.

12. Law MR, Wald NJ, Morris JK, Jordan RE. Value of low dose combination treatment with blood pressure lowering drugs: analysis of 354 randomised trials. Br Med J. 2003;326(7404):1427-1431. Accessed 11 January 2017. 